\title{
Uso de la técnica CLARITY para la identificación de marcadores fluorescentes en cortes gruesos de la médula espinal
}

\author{
Use of the CLARITY technique for identifying fluorescent markers on thick \\ slices of spinal cord
}

Sisti MS ${ }^{1,3 *}$, Nishida Fi,3, ZanuZzi CN ${ }^{1,2,3}$, Portiansky EL ${ }^{1,3}$

1. Laboratorio de Análisis de Imágenes. 2 Cátedra de Histología y Embriología. Facultad de Ciencias Veterinarias, Universidad Nacional de La Plata. 3 Consejo Nacional de Investigaciones Científicas y Técnicas (CONICET). Argentina.

* Correo electrónico de la autora de contacto: mssisti@fcv.unlp.edu.ar

\begin{abstract}
Resumen
El estudio de las interconexiones que establecen las neuronas entre sí o con las células de la glía es uno de los mayores desafíos de la neurociencia. Los anticuerpos fluorescentes permiten la identificación y localización de diferentes estructuras tisulares. Sin embargo, su uso eficiente requiere de la utilización de cortes delgados de muestras, que constituyen una limitación para el estudio de las interconexiones en el sistema nervioso. Asimismo, un mayor espesor de la muestra limita la penetración de la luz emitida por el microscopio, mientras que la opacidad propia del tejido nervioso debida a su alto contenido lipídico dificulta la adquisición de las imágenes al restringir la resolución de los objetos. La técnica CLARITY (del inglés, Clear, Lipid-exchanged, Acrylamide-hybridized Rigid, Imaging/Immunostaining/In situ hybridization-compatible, Tissue hYdrogel) permite subsanar estos inconvenientes. Esta técnica fue adaptada en nuestro laboratorio para el estudio de la médula espinal de rata. De acuerdo con el procedimiento realizado, pudimos obtener un órgano completamente translúcido, estructuralmente intacto y que permitió su procesamiento mediante técnicas de inmunofluorescencia y de lectinhistoquímica sin mayores dificultades. A partir de muestras de más de $1 \mathrm{~mm}$ de espesor se obtuvieron imágenes confocales de gran resolución y de mayor penetrabilidad que las que se obtienen utilizando las técnicas de procesamiento convencionales. La implementación de esta técnica en nuestro laboratorio permitirá optimizar la información obtenida a partir de muestras de interés.
\end{abstract}

\section{Palabras clave}

Fluorescencia, microscopía confocal, procesamiento, análisis de imágenes

\begin{abstract}
The study of the interconnections established between neurons themselves and with glial cells is one of the major challenges of neuroscience. Fluorescent antibodies allow identification and localization of different tissue structures. However, their efficient employment requires the use of thin sections of samples, which constitute a limitation for the study of nervous system interconnections. Likewise, increased thickness of samples limits penetration of the light emitted by the microscope, whereas the opacity of the nervous tissue due to its high lipid content limits the resolution of objects, making the acquisition of images difficult. The CLARITY (Clear, Lipid-exchanged, Acrylamidehybridized Rigid, Imaging/Immunostaining/In situ hybridization-compatible, Tissue hYdrogel) technique makes possible to remedy these disadvantages. This technique was adapted in our laboratory for the study of the rat spinal cord. According to the described procedure we obtained a completely translucid and structurally intact organ that allowed processing through immunofluorescence and lectinhistochemistry techniques without major drawbacks. Confocal images of higher resolution and greater penetrability in comparison with those captured using conventional processing techniques were obtained from more than $1 \mathrm{~mm}$ thick samples. The implementation of this technique in our laboratory will improve the information obtained from our samples of interest.
\end{abstract}

\section{Key words}

Fluorescence, confocal microscopy, processing, image analysis

Fecha de recepción: 27/12/2017

Fecha de aprobación: 27/03/2018
ANALECTA VeT 2018; 38(1): 16-22

Impresa ISSN 03655 14-8 Electrónica ISSN 1514-2590

doi.org/10.24215/15142590eo21 


\section{Introducción}

El estudio de las interconexiones que establecen las neuronas entre sí o con las células de la glía es uno de los mayores desafíos de la neurociencia. El análisis de estas interacciones permitiría comprender, entre otras, las respuestas funcionales que se ven alteradas ante la injuria (Chung \& Deisseroth, 2013).

Para la exploración de las interconexiones se han ensayado, y se continúan ensayando, modelos a diferentes escalas (macro, meso y micro), con gran diversidad de enfoques (Kim et al., 2013). Una aproximación de suma utilidad es la utilización de fluoróforos (moléculas con capacidad de absorber luz, en una determinada longitud de onda y emitirla como luz fluorescente) unidos a anticuerpos que, específicamente, permitan la identificación y localización de moléculas y de estructuras celulares. Para lograr este fin con precisión, es imperioso contar con segmentos delgados de muestra, ya que los fotones, los fluoróforos y los anticuerpos tienen una profundidad de penetración limitada (Chungy Deisseroth, 2013; Kuwajima et al., 2013), convirtiéndose el espesor de la muestra en una gran limitación para las técnicas en las que se los emplean. Por otro lado, para el estudio de las interconexiones, es necesario que las muestras que se desean analizar cuenten con un determinado espesor, definido por la estructura y orientación de las prolongaciones celulares del tejido nervioso. En un corte delgado, serían pocos los detalles de interconexión celular que se podrían apreciar. Con el advenimiento de nuevas técnicas de tinción que permiten teñir cortes de hasta 40 $\mu \mathrm{m}$ de espesor, tales como el free floating (Grabinski et al., 2015), y con la existencia de sistemas de iluminación que penetran más profundamente en los tejidos, tales como los que se obtienen por sistemas de láser continuo o pulsado (Portiansky, 2013), se han mejorado los resultados de las investigaciones en el tejido nervioso y, en consecuencia, su interpretación.

El mayor desafío en el estudio de los sistemas biológicos, mediante técnicas de inmunofluorescencia, es el acceso limitado de los diferentes reactivos a estructuras moleculares presentes en el tejido intacto, debido a que deben atravesar las membranas celulares fosfolipídicas (plasmática y de organelas). Asimismo, la dispersión que se verifica en la interfase lípido/agua determina que el tejido intacto sea opaco, limitando la resolución de la imagen observable al microscopio (Chung et al., 2013).

Teniendo en cuenta las dificultades de la compatibilización entre los efectos del espesor de la muestra, la penetrabilidad de los fluoróforos y de los sistemas de iluminación se han ensayado diversos métodos que permiten convertir diferentes tejidos en muestras transparentes y que, con diferentes ventajas y desventajas, fueron tendiendo a subsanar estos inconvenientes. Uno de los últimos métodos descriptos es el de CLARITY
(Clear Lipid-exchanged Acrylamide-hybridized Rigid Imaging/Immunostaining/In situ hybridization-compatible Tissue-hYdrogel) (Chung et al., 2013). En este sentido, la técnica CLARITY ha emergido como una alternativa superadora dado que permite obtener muestras translúcidas del sistema nervioso, que incluso evitan el seccionamiento físico del tejido. Su fortaleza reside en poder convertir el tejido en un constructo que mantiene su estructura y sus biomoléculas intactas, pero que es ópticamente transparente y, por lo tanto, permite la penetración de los marcadores fluorescentes aún en más de un ciclo de tinción. Mientras que las biomoléculas nativas del tejido se mantienen inalteradas, los lípidos presentes son barridos durante el transcurso de la técnica para la obtención del órgano transparente (Chung et al., 2013).

En el presente trabajo se describe la puesta a punto e implementación de la técnica de CLARITY realizada en nuestro laboratorio, para la determinación de diferentes estructuras nerviosas presentes en la médula espinal de rata.

\section{Materiales y métodos}

\section{Animales}

Se utilizaron 3 ratas macho de la cepa Sprague-Dawley, de entre 3 y 4 meses de edad y con un peso estimado de 150-250 gramos. Los animales fueron provistos por el bioterio del Instituto de Investigaciones Bioquímicas de La Plata (INIBIOLP, Universidad de La PlataCONICET). Las ratas fueron alojadas en un cuarto destinado a este fin, con ciclos de luz-oscuridad de 12:12 h, temperatura controlada $\left(22^{\circ} \mathrm{C}\right)$ y disponibilidad de agua y alimento ad libitum.

Todos los procedimientos llevados a cabo con los animales fueron realizados de acuerdo con las recomendaciones de la Guía para el Cuidado y Uso de Animales de Laboratorio del National Institute of Health (NIH). El protocolo fue aprobado por el CICUAL de la Facultad de Ciencias Veterinarias de la Universidad Nacional de La Plata (C: 45-1-14 T).

\section{Remoción de la médula espinal}

Previo a la eutanasia, los animales se inyectaron con una dosis de ketamina $(40 \mathrm{mg} / \mathrm{Kg}$; IP), sumado a la administración de xilazina ( $8 \mathrm{mg}$ / $\mathrm{Kg}$; IM). A continuación, se perfundieron por vía intracardíaca $100 \mathrm{ml}$ de solución salina tamponada de fosfato (PBS), $\mathrm{pH} 7,4$ a $4{ }^{\circ} \mathrm{C}$, seguida por la administración de una solución tamponada de $4 \%$ paraformaldehído, durante $20 \mathrm{~min}$. La columna vertebral fue removida $y$ fijada en formalina tamponada durante $24 \mathrm{~h}$. Posteriormente, la médula espinal fue disecada, sumergida en una 
solución tamponada de solución criopreservante (30\% sacarosa; $1 \%$ polivinilpirrolidona; 30\% etilenglicol en 1\% PBS 1M y agua destilada para $100 \mathrm{ml}$ ) y almacenada a $-20{ }^{\circ} \mathrm{C}$ hasta su utilización.

Técnica de CLARITY para la clarificación de la médula espinal

Para la implementación de la técnica se recurrió a una modificación de la técnica original formulada por Chung et al. (2013). En el presente trabajo se prescindió de la electroforesis estipulada originalmente, basados en los trabajos de Tomer et al. (2014) y Zheng y Rinaman (2015). Para realizar la técnica, la médula espinal fue incubada durante 3 días, a $4{ }^{\circ} \mathrm{C}$ en un tubo de $20 \mathrm{ml}$ con una solución de $4 \%$ paraformaldehído, $4 \%$ acrilamida $\left(\mathrm{C}_{3} \mathrm{H}_{5} \mathrm{NO}\right), 0,05 \%$ bis-acrilamida y 0,025 \% del catalizador VA044, todo ello en 10x-PBS (Tomer et al., 2014). Pasado el tiempo, se le agregó aceite comestible de girasol, hasta colmar su capacidad. De esta manera, se expulsó todo el oxígeno contenido en el tubo, con el propósito de evitar la interferencia en la acción catalítica del VAo44. Seguidamente, se ubicó verticalmente el tubo en una grilla sumergida en un baño agitador (EYELA Thermopet NTT-220, Tokyo Rikakikai Co Ltd, Japón) con temperatura controlada de $37{ }^{\circ} \mathrm{C}$, durante $4 \mathrm{~h}$, hasta que se verificó la gelificación de la solución contenida en el tubo, con la médula espinal incluida en él. Una vez obtenido el gel, se extrajo el órgano del tubo y se lo separó del resto del gel con ayuda de una espátula. A continuación, fue colocado en otro tubo que contenía una solución de lavado, constituida por $20 \%$ dodecilsulfato de sodio $\left(\mathrm{C}_{12} \mathrm{H} 25 \mathrm{NaO}_{4} \mathrm{~S}-\mathrm{SDS}\right)$ en tampón de ácido bórico $\left(\mathrm{H}_{3} \mathrm{BO}_{3}\right)$ 1M, pH 8,5 (Tomer et al., 2014). Se colocó nuevamente en el agitador a $37^{\circ} \mathrm{C}$, con agitación constante. Se realizaron dos lavados, de $24 \mathrm{~h}$ cada uno. Finalizados estos lavados, se continuó con el proceso de clarificación incubando la muestra en la solución de SDS y recambiando la solución de SDS cada $48 \mathrm{~h}$ hasta obtener el órgano translúcido (Fig. 1A). La duración aproximada total del proceso de clarifica-ción fue de 6 semanas. Pasado este tiempo, el órgano se extrajo y se colocó en un nuevo tubo con solución PBST (PBS + 0,1\% Tween 20) para lavar el exceso de SDS, durante $48 \mathrm{~h}$, cambiando la solución cada 24 h, con agitación y temperatura constante $\left(37^{\circ} \mathrm{C}\right)$.

Transcurridas las $48 \mathrm{~h}$, se procedió al seccionamiento de la región de interés (segmento $\mathrm{C}_{5}$ ) a partir del órgano entero, con la ayuda de una hoja de bisturí. La muestra obtenida se conservó en solución PBST hasta su procesamiento.

\section{Técnica de inmunofluorescencia}

La sección de interés fue colocada en un tubo Eppendorf y lavada con solución PBST duran- te $48 \mathrm{~h}$, con recambio de la solución a las $24 \mathrm{~h}$. Seguidamente, la sección se incubó con el anticuerpo monoclonal anti-neurofilamento (anti-NF, ready to use, Dako Cytomation, Carpinteria, EE.UU.) durante $48 \mathrm{~h}$, a $37{ }^{\circ} \mathrm{C}$ y con agitación constante. Este anticuerpo se utiliza para identificar neurofilamentos, los que se encuentran presentes en las proyecciones axonales de las neuronas. Posteriormente, se lavó con solución PBST durante $24 \mathrm{~h}$ a $37{ }^{\circ} \mathrm{C}$ y con agitación constante. Finalizado el lavado, la muestra fue incubada con el anticuerpo secundario (AlexaFluor ${ }^{\circledR}$ 488, Invitrogen, EE.UU.) durante $48 \mathrm{~h}$, lavada con PBST durante $24 \mathrm{~h}$ y, finalmente, incubada con DAPI ( 4 ', 6-diamino-2-fenilindol) 1:1000 durante otras 48 $\mathrm{h}$, para la identificación de núcleos. Por último, se lavó la muestra con PBST durante $24 \mathrm{~h}$ y se la incubó con glicerol como medio de montaje (87\%, Merck Química Argentina S.A.I.C, Buenos Aires, Argentina) durante otras $24 \mathrm{~h}$.

\section{Técnica de lectinhistoquímica fluorescente}

La sección de interés fue colocada en un tubo Eppendorf y lavada con solución PBST (PBS $+0,1 \%$ Tween 20) durante $48 \mathrm{~h}$, con recambio de la solución a las $24 \mathrm{~h}$. Seguidamente, se la incubó con la lectina fluoresceinada Pisum sativum (PSA) (Fluorescein Pisum Sativum Agglutinin, Vector Laboratories Inc., Burlingame, EE.UU.) (dilución 30:1000), que se une específicamente a residuos de $\alpha$-D-manosa y $\alpha$-D-glucosa, a $4{ }^{\circ} \mathrm{C}$, durante $48 \mathrm{~h}$. Pasado ese tiempo, se lavó nuevamente durante 24 h con solución PBST, antes de incubarla con glicerol como medio de montaje, durante otras $24 \mathrm{~h}$.

\section{Montaje de la muestra y captura de imágenes}

Las secciones sometidas a las técnicas de tinción descritas fueron colocadas en un portaobjetos especialmente diseñado en nuestro laboratorio (Fig. 1B). Este dispositivo, confeccionado con acrílico, tiene $1 \mathrm{~mm}$ de espesor y un orificio central para albergar la muestra. Una vez colocada la muestra, el orificio central fue rellenado con el medio de montaje. Ambas caras del portaobjetos fueron selladas con sendos cubreobjetos. La muestra fue conservada al abrigo de la luz y a $4{ }^{\circ} \mathrm{C}$, hasta el momento de la captura de imágenes multidimensionales. La captura se llevó a cabo utilizando un microscopio confocal (Olympus FV100o, Japón). Las imágenes multidimensionales capturadas fueron procesadas para lograr imágenes bidimensionales, mediante el algoritmo "máxima intensidad" de un programa informático para el análisis digital de imágenes (cellSens Dimension, v1.7 - Olympus). 


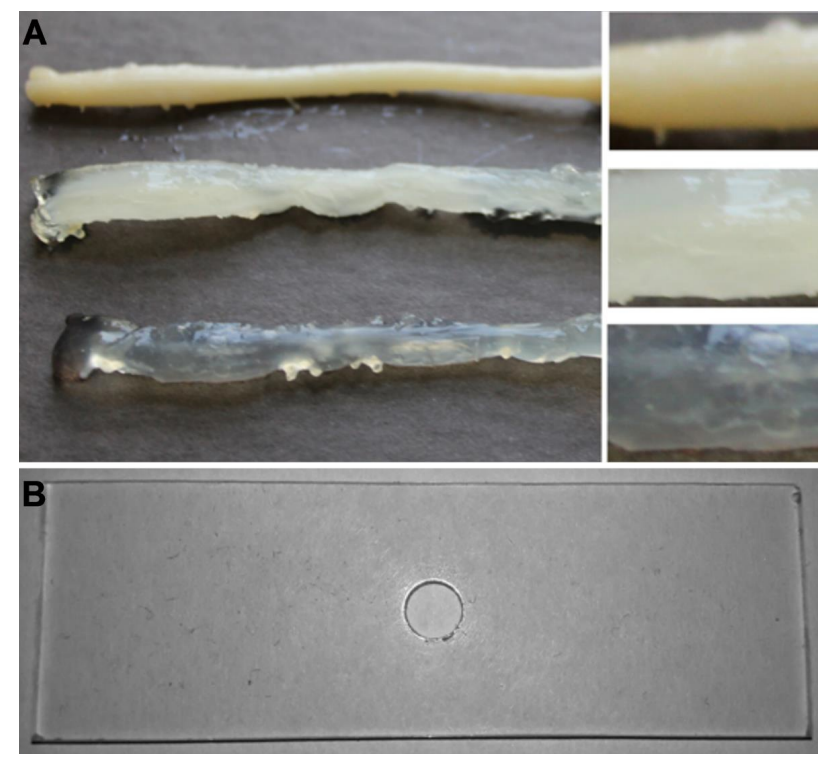

Figura 1. A- Se muestra la progresión del proceso de clarificación. De arriba hacia abajo: el órgano perfundido y extraído del animal, el mismo órgano luego de 4 semanas de proceso de clarificación y luego de 6 semanas. Recuadros: detalle de un sector de cada una de las 3 etapas. B- Imagen del portaobjetos diseñado en nuestro laboratorio para la contención/montaje de la muestra.
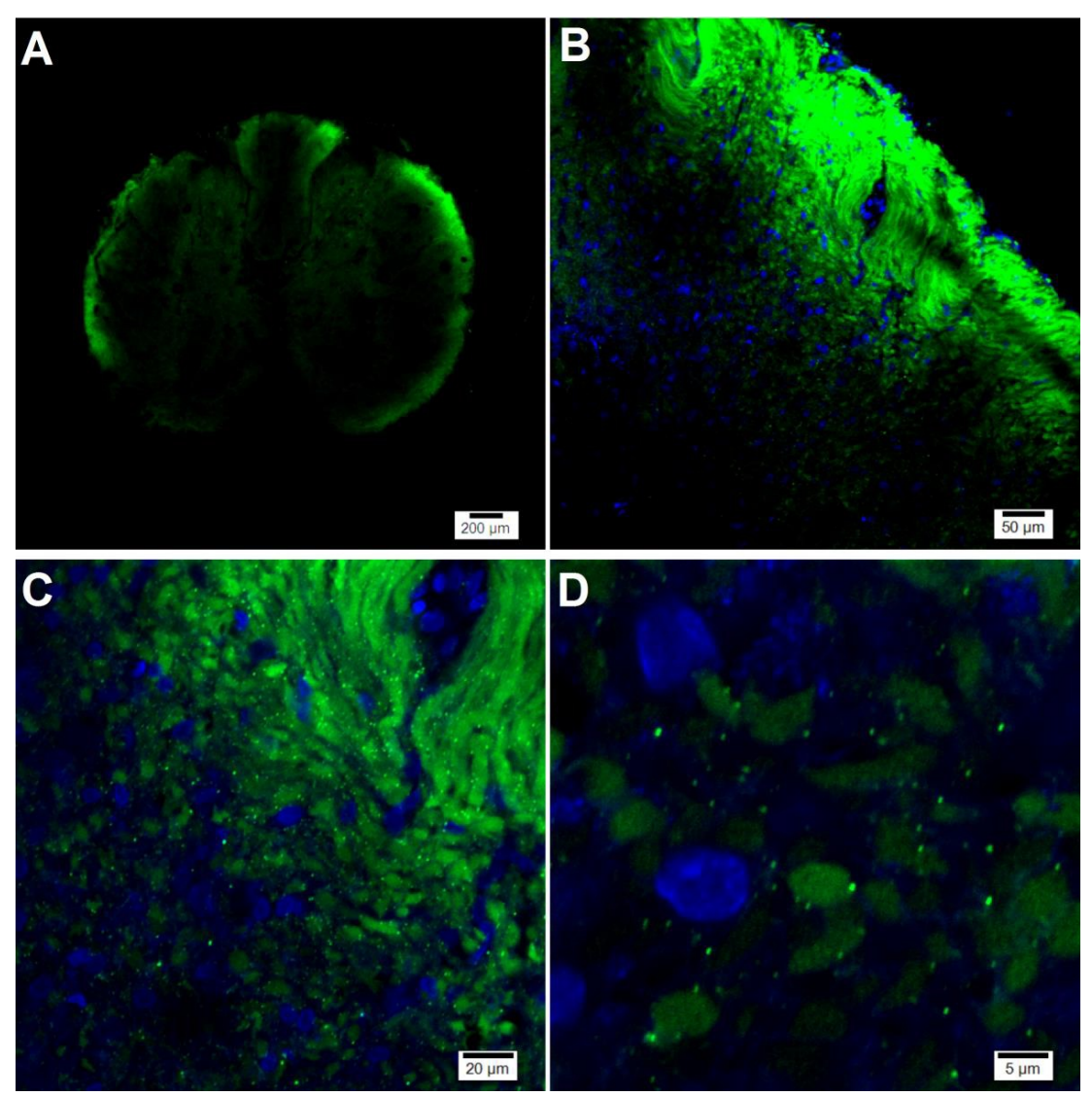

Figura 2. Inmunofluorescencia de una sección del segmento $\mathrm{C}_{5}$ de la médula espinal clarificada. A- Imagen bidimensional que muestra una vista panorámica del segmento $\mathrm{C}_{5}$, en donde se aprecia la tinción (verde) correspondiente a los neurofilamentos de los axones neuronales, tanto en la sustancia blanca como en la sustancia gris. B- Mayor aumento de la imagen, donde se observan los haces nerviosos penetrando en el asta dorsal de la médula. Para obtener la imagen multidimensional se capturaron 20 imágenes bidimensionales con un espaciado de $3 \mu \mathrm{m}$ entre ellas. Las estructuras que reflejan el azul corresponden a núcleos de neuronas o células de la glía. C- Detalle de la imagen observada en B. D- detalle, a mayor aumento, de cortes transversales de axones que contienen neurofilamentos. 


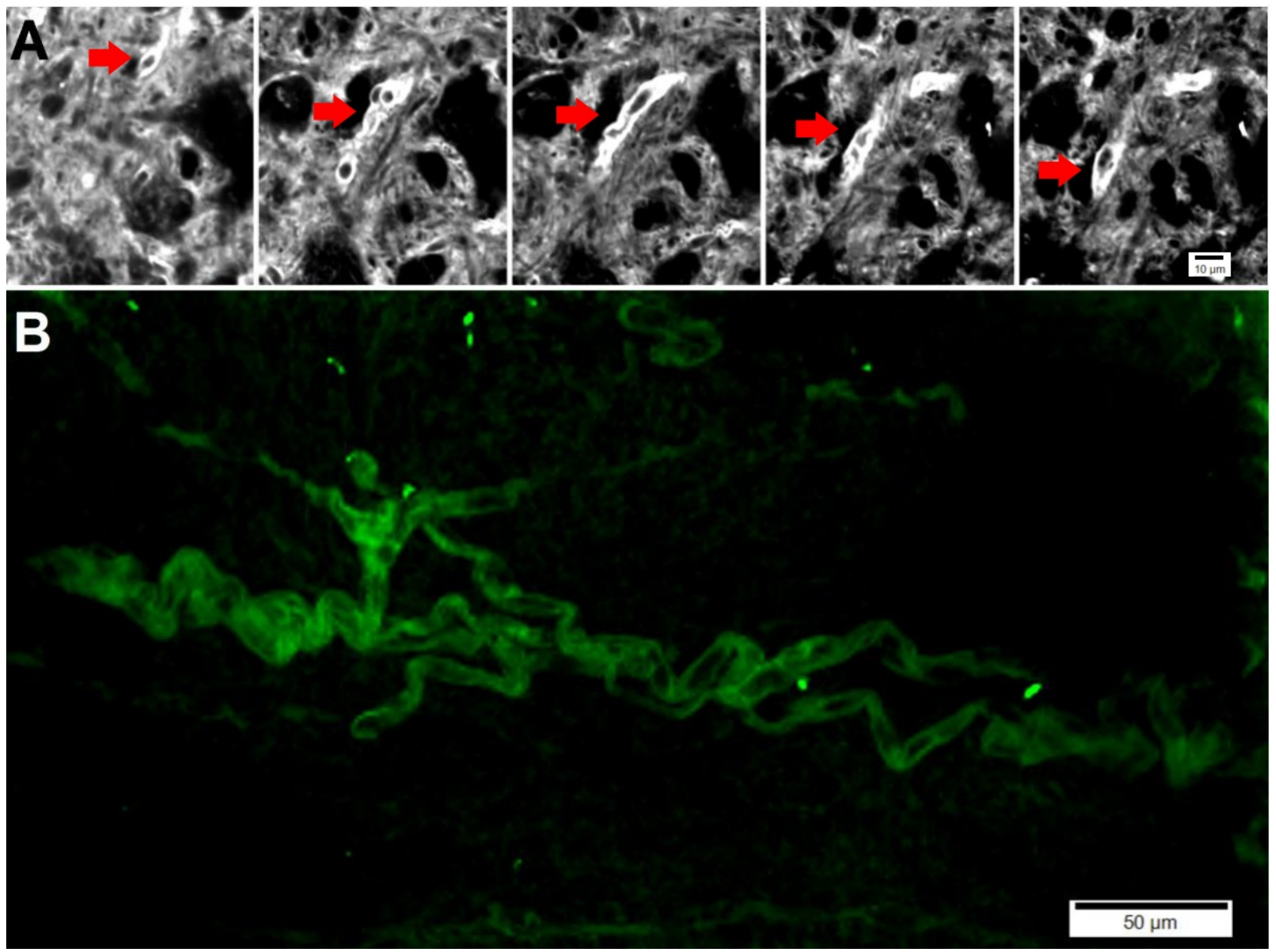

Figura 3. Tinción lectinhistoquímica-fluorescente del segmento $\mathrm{C}_{5}$ de la médula espinal clarificada. A. Imágenes bidimensionales en las que se observan vasos sanguíneos en diferentes secciones ópticas en el espesor de la muestra (flechas). B. Reconstrucción tridimensional (renderización) de vasos sanguíneos en el espesor de la muestra, con sus correspondientes ramificaciones. Para la reconstrucción de la imagen 3D se utilizaron 40 imágenes bidimensionales apiladas (stack - imagen multidimensional), separadas $3 \mu \mathrm{m}$ entre ellas.

\section{Resultados}

\section{Técnica de inmunofluorescencia}

La observación al microscopio confocal de la sección incubada con el anticuerpo anti-NF permitió identificar las proyecciones axonales, tanto en la periferia del órgano (ingreso y egreso de fibras nerviosas), como en el interior de la sustancia gris, con una distribución longitudinal. La tinción con DAPI permitió visualizar todos los núcleos presentes en el espesor de la sección de la médula espinal (Fig. 2).

\section{Técnica de lectinhistoquímica}

Mediante el microscopio confocal, fue posible observar la fluorescencia emitida por la lectina fluoresceinada PSA asociada al recorrido de diferentes vasos sanguíneos (Fig. 3).

\section{Discusión y conclusiones}

Aunque la utilización de marcadores fluorescentes es de gran utilidad para la obtención de información, no siempre es posible identificar las diversas moléculas en el tejido. Entre otras razones, cabe mencionar que las muestras fijadas en paraformaldehído limitan el acceso de los anticuerpos fluorescentes a los diversos epitopes (Ke et al., 2013). Por otro lado, la opacidad de las muestras limita la profundidad de acceso de los fotones emitidos por la fuente de luz del microscopio, ya sea por difracción de la luz como por su absorción dentro de la muestra (Orlich \& Kiefer, 2017). Esta opacidad reduce la resolución de los objetos presentes en la muestra. La opacidad del material se debe, principalmente, a la presencia de lípidos como parte constitutiva del tejido, los que pueden enmascarar la expresión de los anticuerpos fluorescentes, aún bajo apropiados sistemas de iluminación (Chung et al., 2013).

Para tratar de eliminar el efecto opalescente que ejercen los lípidos se realizaron 
múltiples ensayos. Entre otros, se mencionan aquellos que intentaron reducir las diferencias en los índices de refracción de las muestras. Para ello, las muestras fueron deshidratadas y posteriormente incubadas con solventes orgánicos, de modo que el índice de refracción resultante fuese similar al de las membranas lipídicas, intentando, de ese modo, reducir la dispersión de la luz. Sin embargo, a pesar de permitir la transparencia del órgano, estos métodos provocaron a una escasa supervivencia de los marcadores fluorescentes (Kim et al., 2013). Esta limitación se contrarrestó parcialmente gracias al método 3DISCO, que logró extender la supervivencia de la señal fluorescente hasta 2 días, pero sólo para muestras de tamaño reducido (Ertürk y Bradke, 2013). Asimismo, otro método denominado Scale, permitió la obtención de muestras trans-parentes que conservaban la fluorescencia por un tiempo mayor. Pese a ello, el tejido nervioso se mantuvo opaco, aun cuando se incubaba durante semanas o meses, y experimentaba expansión de su volumen (Orlich \& Kiefer, 2017). El método de Clear $T$, desarrollado posteriormente, logró la transparencia de un embrión de ratón sin expansión del tejido (Kuwajima et al., 2013). Sin embargo, su aplicación en muestras de mayor tamaño aún no se ha establecido. Finalmente, el método SeeDB permitió la obtención de muestras transparentes y la conservación de la fluorescencia, aunque para el procesamiento de muestras de mayor tamaño se requería del uso de altas temperaturas, lo que conllevaba, inevitablemente, a una pérdida de la señal fluorescente (Ke et al., 2013).

Si bien la técnica CLARITY demanda un mayor tiempo en comparación con otros métodos, es ampliamente superadora en cuanto a profundidad y calidad de las imágenes que pudieran obtenerse al emplearla. Mientras que la profundidad estimada de penetración de los fotones para la microscopía confocal ronda los $150 \mu \mathrm{m}$ desde la superficie (Kim et al., 2013), con el uso de CLARITY se logra un mayor alcance, obteniendo imágenes de excelente calidad. Si bien con los microscopios de láser pulsado se obtienen imágenes a partir de muestras de varios cientos de micrómetros de espesor (Tomer et al., 2014), se requiere de anticuerpos específicos y muy costosos y una puesta a punto muy rigurosa de la técnica, más allá de la posibilidad de contar o acceder a ese tipo de microscopio.

Por lo general, para la observación microscópica se utilizan secciones pequeñas de tejido, que son procesadas para su inclusión y posterior corte. Mediante la implementación de esta técnica, pudimos procesar el órgano entero sin alterarlo macroscópicamente. Una vez obtenida la transparencia deseada, fue seccionado (segmento $\mathrm{C}_{5}$ ), procesado mediante las técnicas de inmunofluorescencia y lectinhistoquímica fluorescente, montado y observado al microscopio. Ninguno de los pasos mencionados generó algún tipo de inconveniente en la integridad estructural.
Además, al haber empleado una técnica que difiere de aquella formulada originalmente (Chung et al., 2013) se obtuvo una muestra traslúcida y carente de la tonalidad amarillenta que adquieren los órganos como resultado de la aplicación del campo eléctrico (Lee et al., 2014), lo cual redundó en una mayor transparencia, de acuerdo con lo que se menciona en la literatura. Cabe mencionar que este mismo estudio hubiese sido imposible de realizar en los mismos segmentos medulares que no hubiesen sido procesados mediante el método descripto. El único inconveniente del empleo de la técnica de CLARITY es la limitada preservación de la muestra, debido a los procesos de descomposición que se inician, aun a bajas temperaturas, a diferencia de lo que ocurre con muestras procesadas de manera rutinaria.

En el presente trabajo se describe un método vanguardista, simple y sin requerimiento de equipamiento excesivamente sofisticado. Se logró la observación de muestras con alta nitidez y poco fondo (background), lo que redundó en una mayor resolución de las estructuras marcadas. La incorporación de esta técnica a los métodos utilizados rutinariamente en nuestro laboratorio permitirá optimizar la información obtenida a partir de las muestras de interés.

\section{Agradecimientos}

El presente trabajo fue financiado por la Agencia Nacional de Promoción Científica y Tecnológica (ANPCyT), Ministerio de Ciencia, Tecnología e Innovación Productiva de la Nación (MINCyT), PICT-2012-0574.

\section{Conflicto de intereses}

Todos los autores declaran que no existe conflicto de intereses, incluyendo entre estos últimos las relaciones financieras, personales o de otro tipo con otras personas u organizaciones que pudieran influir de manera inapropiada en el trabajo. conflicto de intereses que declarar.

\section{Bibliografía}

Chung K, Wallace J, Kim S-Y, Kalyanasundaram S, Andalman AS, Davidson TJ, Mirzabekov JJ, Zalocusky KA, Mattis J, Denisin AK, Pak S, Bernstein H, Ramakrishnan C, Grosenick L, Gradinaru V, Deisseroth K. 2013. Structural and molecular interrogation of intact biological systems. Nature. 497(7449):332-7.

doi: $10.1038 /$ nature12107

Chung K y Deisseroth K. 2013. CLARITY for mapping the nervous system. Nature Methods. 
2013 Jun;10(6):508-13.

doi: $10.1038 /$ nmeth.2481

Ertürk A y Bradke F. 2013. High-resolution imaging of entire organs by 3 -dimensional imaging of solvent cleared organs (3DISCO). Experimental Neurology. 242:57-64.

doi: 10.1016/j.expneurol.2012.10.018

Grabinski TM, Kneynsberg A, Manfredsson FP, Kanaan NM. 2015. A method for combining RNAscope in situ hybridization with immunohistochemistry in thick free-floating brain sections and primary neuronal cultures. PLoS One. 10(3):e0120120.

doi: 10.1371/journal.pone.0120120

Ke M-T, Fujimoto S, Imai T. 2013. SeeDB: a simple and morphology-preserving optical clearing agent for neuronal circuit reconstruction. Nature Neuroscience. 6(8):1154-61.

doi: $10.1038 / \mathrm{nn} .3447$

Kim S-Y, Chung K, Deisseroth K. 2013. Light microscopy mapping of connections in the intact brain. Trends Cognitive Sciences. 17(12):596-9.

doi: 10.1016/j.tics.2013.10.005

Kuwajima T, Sitko AA, Bhansali P, Jurgens C, Guido W, Mason C. 2013. ClearT: a detergent- and solvent-free clearing method for neuronal and nonneuronal tissue. Development. 140(6):1364-8.

doi: 10.1242/dev.091844
Lee H, Park J-H, Seo I, Park S-H, Kim S. 2014. Improved application of the electrophoretic tissue clearing technology, CLARITY, to intact solid organs including brain, pancreas, liver, kidney, lung, and intestine. BMC Developmental Biology. 21;14:48.

doi: 10.1186/s12861-014-0048-3

Orlich M, Kiefer F. 2017. A qualitative comparison of ten tissue clearing techniques. Histology \& Histopathology. 12:11903.

doi: 10.14670/HH-11-903

Portiansky EL. 2013. Análisis multidimensional de imágenes digitales. La Plata, Argentina. Universidad Nacional de La Plata, http:// hdl.handle.net/10915/48218.

Tomer R, Ye L, Hsueh B, Deisseroth K. 2014. Advanced CLARITY for rapid and high-resolution imaging of intact tissues. Nature Protocols. 9(7): 1682-97.

doi: $10.1038 /$ nprot.2014.123

Zheng H y Rinaman L. 2015. Simplified CLARITY for visualizing immunofluorescence labelling in the developing rat brain. Brain Structure \& Function. 221(4):2375-83.

doi: 10.1007/s00429-015-1020-0 